\title{
Design and Optimization of a Radial Flow Heat Sink under Free Convection at Steady State Condition
}

\author{
M. M. Rahman*, a, H. Bhowmik ${ }^{\text {b }}$, S. Talukdar ${ }^{\text {b }}$ \\ ${ }^{a}$ Department of Mechanical Engineering, University of Saskatchewan, Canada \\ ${ }^{\mathrm{b}}$ Department of Mechanical Engineering, DUET, Gazipur, Bangladesh
}

\begin{abstract}
Three dimensional numerical models were developed to make prediction free convection heat transfer at steady state condition from radial flow heat sink. The air was considered as the medium of heat transfer. In radial flow heat sink, heat conducts through base in radial pattern and is uniformly transported to the fins. The Tagucy method was used to investigate the effect of several design parameters such as fin length, fin height, number of fins and heat sink base radius on heat transfer. There are five factors and four levels on each factor were chosen. Sixteen types of model were analyzed to obtain total heat transfer for each model. The result was used to estimate the optimum designed values of the parameters affecting the heat sink efficiency. The reproducibility of the optimum design value was verified. The average rate of heat transfer of optimum model was increased by more than $50 \%$ than the reference model. Finally, the heat transfer data of radial flow heat sink were correlated of several outer radius by an equation.
\end{abstract}

Keywords: Natural convection, Laminar flow, Radial flow, Heat transfer, Optimization

\section{Introduction}

Natural convection heat transfer from finned surface is used for the thermal management of low power-density devices. Radial flow heat sink with finned surfaces has been extensively used in many applications. These types of heat sinks can be a very successful solution for LED application and processor based desktop computer. The heat sink with radial fin orientation provides uniform temperature distribution at equilateral distances from the base, which enhances the thermal performance. All these imported issues provided motivation of the present study.

The performance analysis and optimization of conventional heat sinks were studied by many researchers both experimentally and numerically [17]. A theoretical and experimental study was conducted by Kobus and Oshio [1], to study the effect of various geometries on thermal resistance of the heat sink. In the natural convection region, the author obtained the improved performance at the increased fin length, while the fin spacing was constant. Huang et al. [2] investigated the orientation effect of square pin fin on natural convection heat transfer and obtained the high heat transfer coefficient at the upward and sideward facing orientation. Zografos and Sunderland [3] analyzed the heat transfer performance of inline and staggered pin fins and obtained better performance from inline

${ }^{*}$ Corresponding author.

E-mail: mdr972@mail.usask.ca; mostafizme@gmail.com

(C) 2016 International Association for Sharing Knowledge and Sustainability

DOI: $10.5383 /$ ijtee.13.02.001 
fins compared to the staggered fins. Sparrow and Vemuri [4] investigated the effect of heat transfer on the fin orientation and obtained the highest heat transfer coefficient at the upward facing orientation. The authors also optimized the fin density and height. Inada et al. [5] investigated experimentally the effects of vertical fins with constant height on heat transfer rate. The authors also investigated the effects of flow orientation and obtained an enhanced heat transfer rate at the upward flows.

As this survey shows, many of the analyses were based on the heat transfer from the rectangular plate fin heat sink, and completely ignored the transfer of heat for the radial fluid flow conditions and optimization of such types of heat sink. The present analysis simulated the natural convection heat transfer from an array of aluminum rectangular fins that were oriented radially on a horizontal circular base plate. Parametric studies were performed in order to determine the optimum heat sink. The optimum design value of each parameter was presented, and the reproducibility of the results was discussed. Finally, the heat transfer data at different outer radius were correlated.

\section{Description of the Geometry}

\section{Geometric dimension of the heat sink}

The radial flow heat sink with rectangular fins is arrayed as shown in Figure 1. The baseline design consisted of long, medium and short fins, arranged radially along the flow direction. To increase the surface area for heat transfer, compared to the case with only long fins, the medium and short fins were introduced. The heat sink base and fins were made of Aluminum of thermal conductivity, $202.4 \mathrm{~W} /(\mathrm{m} \cdot \mathrm{K})$. The considered heat transfer medium was air $(\mathrm{Pr}=$ $0.7)$. Heat sink geometry was described by the fin lengths $L_{\ell}, L_{m}, L_{s}$, fin height $H$, fin thickness $t$, heat sink base thickness $t_{b}$ and the angle between two long fins $\theta$. The $H, t, t_{b}$ were chosen as constant, i.e. $H=$ $21.3 \mathrm{~mm}, t=t_{b}=2 \mathrm{~mm}$. The inner radius $\left(R_{i}\right)$ was kept constant as $10 \mathrm{~mm}$ and the outer radius $\left(R_{o}\right)$ of the heat sink varied from $75 \mathrm{~mm}$ to $115 \mathrm{~mm}$. The surrounding air temperature was maintained constant as $303 \mathrm{~K}$. The base temperature of the heat sink was varied from $330 \mathrm{~K}$ to $350 \mathrm{~K}$.

\section{Modeling Approach and Governing Equations}

The modeling was approached based on the assumptions, i.e. the flow velocity was steady and laminar natural convection [6], Radiation heat loss was neglected [7], all the properties of air except density were assumed constant and the properties were assumed constant for the fin material, Aluminum.

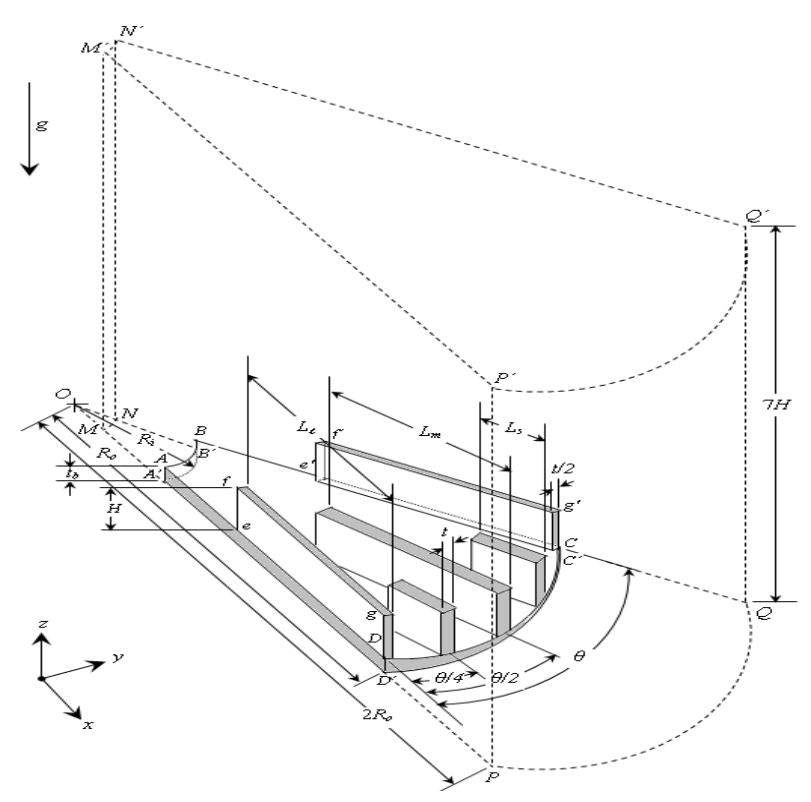

Fig.1: Schematic diagram of the heat sink

Three dimensional steady-state laminar flow model was selected to simulate the flow and heat transfer from the heat sink. The CFD package FLUENT, based on finite-volume method was employed to solve the fluid flow and heat transfer problems. A segregated solver was used to work out the governing integral equations for the conservation of mass, momentum, and energy. The preprocessor GAMBIT was used to create a computational geometry. After specifying the geometry, ANSYS ICEM was used for the boundary conditions and to create the computational mesh. The meshes were then exported to the software FLUENT to carry out the numerical simulation. The finite volume technique and the semi-implicit method for pressurelinked equation (SIMPLE) were used to solve the basic conservation Eqs. (1) to (5), in three dimensional Cartesian coordinates.

The continuity equation:

$$
\frac{\partial \rho}{\partial t}+\nabla \cdot(\rho u)=0
$$

The momentum equation in the $x, y, z$ directions:

$$
\frac{\partial\left(\rho u^{2}\right)}{\partial x}+\frac{\partial(\rho u v)}{\partial y}+\frac{\partial(\rho u w)}{\partial z}=-\frac{\partial P}{\partial x}+\mu\left(\frac{\partial^{2} u}{\partial x^{2}}+\frac{\partial^{2} u}{\partial y^{2}}+\frac{\partial^{2} u}{\partial z^{2}}\right)
$$


$\frac{\partial(\rho v u)}{\partial x}+\frac{\partial\left(\rho v^{2}\right)}{\partial y}+\frac{\partial(\rho v w)}{\partial z}=-\frac{\partial P}{\partial y}+\mu\left(\frac{\partial^{2} v}{\partial x^{2}}+\frac{\partial^{2} v}{\partial y^{2}}+\frac{\partial^{2} v}{\partial z^{2}}\right)$

$\frac{\partial(\rho w u)}{\partial x}+\frac{\partial(\rho w v)}{\partial y}+\frac{\partial\left(\rho w^{2}\right)}{\partial z}=-\frac{\partial P}{\partial z}+\mu\left(\frac{\partial^{2} w}{\partial x^{2}}+\frac{\partial^{2} w}{\partial y^{2}}+\frac{\partial^{2} w}{\partial z^{2}}\right)+g\left(\rho-\rho_{a}\right)$

The energy equation:

$\frac{\partial(\rho u T)}{\partial x}+\frac{\partial(\rho v T)}{\partial y}+\frac{\partial(\rho w T)}{\partial z}=\frac{k_{f i n}}{c_{p}}\left(\frac{\partial^{2} T}{\partial x^{2}}+\frac{\partial^{2} T}{\partial y^{2}}+\frac{\partial^{2} T}{\partial z^{2}}\right)$

For the fin material, temperature distribution was modeled by Fourier's heat conduction equation:

$\left(\frac{\partial^{2} T}{\partial x^{2}}+\frac{\partial^{2} T}{\partial y^{2}}+\frac{\partial^{2} T}{\partial z^{2}}\right)=0$

For an incompressible flow, the solver computed the density according to the ideal gas law:

$\rho=\frac{p}{\frac{R_{c}}{M_{w}} T_{a}}$

\subsection{Boundary conditions}

Figure 1 illustrates the computational domain, where the fin base surface $\mathrm{A}^{\prime} \mathrm{B}^{\prime} \mathrm{C}^{\prime} \mathrm{D}^{\prime} \mathrm{A}^{\prime}$ was held at constant temperature, $T_{\text {base }}$ and constant heat flux, $q^{\prime \prime}$. The surrounding air temperature was assumed constant, $T_{a}$. The Periodic boundary conditions were applied at the surfaces $\mathrm{ADD}^{\prime} \mathrm{A}^{\prime} \mathrm{A}$, efgDe, MA'AefgDD'PP'M'M, and at the back BCC'B'B, $\mathrm{e}^{\prime} \mathrm{f}^{\prime} \mathrm{g}^{\prime} \mathrm{Ce}^{\prime}, \quad \mathrm{NB}{ }^{\prime} \mathrm{Be}^{\prime} \mathrm{f}^{\prime} \mathrm{g}^{\prime} \mathrm{CC}^{\prime} \mathrm{QQ} \mathrm{Q}^{\prime} \mathrm{N}^{\prime} \mathrm{N}$. FLUENT treated the flow at a periodic boundary as though the opposing periodic plane was a direct neighbor of the cells adjacent to the first periodic boundary. The Pressure inlet boundary condition was applied at the surfaces MNB'A 'M, D'PQC'D', and PQQ'P'P, and the pressure outlet boundary condition was applied at the surfaces $\mathrm{M}^{\prime} \mathrm{P}^{\prime} \mathrm{Q}^{\prime} \mathrm{N}^{\prime} \mathrm{M}^{\prime}$ and $\mathrm{MNN}^{\prime} \mathrm{M}^{\prime} \mathrm{M}$.

\subsection{Calculation of numerical data}

The thermo physical properties were evaluated at the film temperature, $T_{f}=\left(T_{\text {base }}+T_{a}\right) / 2$.

The total heat transfer, $q_{T}$ from the rectangular fins and un-finned base was calculated as

$q_{T}=h_{\text {avg }}\left(A_{\text {fin }}+A_{b}\right) \theta_{T}$

where, $A_{f i n}$ and $A_{b}$ were the fin area and base area, available for heat transfer. The $A_{\text {fin }}$ and $A_{b}$ were calculated as

$$
\begin{aligned}
& A_{f i n}=\left(n_{\ell} L_{\ell}+n_{m} L_{\mathrm{m}}+n_{s} L_{\mathrm{s}}\right)(t+2 H)+2 H t\left(n_{\ell}+n_{m}+n_{s}\right) \\
& A_{b}=\pi\left(R_{o}{ }^{2}-R_{i}^{2}\right)+2 \pi t_{b}\left(R_{o}+R_{i}\right)-t\left(n_{\ell} L_{\ell}+n_{m} L_{\mathrm{m}}+n_{s} L_{\mathrm{s}}\right)
\end{aligned}
$$

The number of fins was calculated as

$$
n_{\ell}=n_{m}=n_{s} / 2=360^{\circ} / \theta
$$

\section{Results and Analysis}

\subsection{Model validation}

Numerical results of the reference model, $\theta=18^{\circ}, R_{o}$ $=75 \mathrm{~mm}, L_{\ell}=55 \mathrm{~mm}, L_{m}=40 \mathrm{~mm}, L_{s}=15 \mathrm{~mm}$, was compared with experimental data. The experiment was performed at the surrounding air temperature of $T_{a}=292.4 \mathrm{~K}$. The constant heat flux, $q^{\prime \prime}=371.6$ $\mathrm{W} / \mathrm{m}^{2}$ was maintained at the base of the heat sink. Four type- $T$ thermocouples were attached on the base of the heat sink to measure temperatures at different points. The average of the four temperatures was yielded the temperature of the heat sink. It was obtained that the difference between the experimental and numerical result was $1.74^{\circ} \mathrm{C}$ that showed a reasonably good agreement. However, our model was definitely able to reproduce qualitative data, as will be described in the following sections.

\subsection{Optimization of geometric parameters}

For the present radial flow heat sink, the heat transfer rate depends mainly on the diameter of the heat sink, number of fins and the lengths of the long, medium and short fins. The effects of geometric parameters, namely outer radius of the heat sink, number of fins, lengths of the long, medium, short fins on heat sink performance were analyzed simultaneously using the Taguchi method [8]. The orthogonal array (OA) was adopted for the scheme design, of which the schemes of experiments were determined. For the present study, four geometric parameters as $\mathrm{A}, \mathrm{L}, \mathrm{M}$ and $\mathrm{S}$ were promoted as control factors, and each control factor having four levels as listed in Table 1. As for orthogonal array method, the orthogonal table $L_{16}\left(4^{4}\right)$ needed 16 test cases, which was based on four factors and four levels on each factor. The average heat transfer coefficient, $h_{\text {avg }}$ data were obtained from the simulation calculation for the each sample. Then the heat transfer rate, $q_{T}$ was calculated according to Eq. (8).

In order to select the levels and control factors more efficiently, the heat transfer data were converted into the signal-to-noise ( $\mathrm{SN}$ ) ratio [9]. A larger SN ratio was preferred and was defined by the following equation representing the static characteristics [10]

$\mathrm{SN}=-10 \log _{10}\left[\frac{1}{N} \sum_{i=1}^{N} \frac{1}{\left(q_{T}\right)_{i}^{2}}\right]$ 
Here, $N$ is the number of design point, i.e. $N=1$. The SN ratios for sixteen cases were obtained based on the outer radius, $R_{o}=75,95,115 \mathrm{~mm}$. The factorial effect and contribution ratio of each factor from those $\mathrm{SN}$ ratios were calculated. The $\mathrm{SN}$ ratios of levels on each factor in Table 2 , for $R_{o}=75 \mathrm{~mm}$ were calculated from the arithmetic average of $\mathrm{SN}$ ratios corresponding to each level [11].

The contribution ratio $(\mathrm{CR})$ means the effect of each factor on heat transfer rate, $q_{T}$, i.e. the performance characteristics of a heat sink. This was calculated using $\mathrm{D}$ that indicated the difference between maximum and minimum SN ratios on each factor. The contribution ratio of each factor was obtained from the ratio of the D corresponding to each factor to the total D [11]. For example, the effect of each factor on heat transfer rate was obtained as $81.24 \%$ for angle $\mathrm{A}$, and $3.61 \%, 1.22 \%, 13.93 \%$ for $\mathrm{L}, \mathrm{M}, \mathrm{S}$, respectively, for $R_{o}=75 \mathrm{~mm}$ as presented in Table 2. It was also found that the contribution ratio of factor A decreased with increasing radius $R_{o}$, whereas the contribution ratios of factors $\mathrm{L}, \mathrm{M}, \mathrm{S}$ increased with increasing radius $R_{o}$. Thus, all these factors significantly affected the heat transfer performance of the heat sink.

Table 1: Levels of each control factor in this study

\begin{tabular}{llccccc}
\hline \multirow{2}{*}{ Factors } & \multirow{2}{*}{$\begin{array}{c}\text { Base } \\
\text { model }\end{array}$} & \multicolumn{4}{c}{ Levels of each factor } \\
\cline { 4 - 7 } & & & L-1 & L-2 & L-3 & L-4 \\
\hline $\mathrm{A}$ & Angle, $\theta$ & 18 & 12 & 15 & 18 & 20 \\
$\mathrm{~L}$ & $L_{\ell} / R_{o}$ & 0.733 & 0.65 & 0.7 & 0.75 & 0.8 \\
$\mathrm{M}$ & $L_{m} / L_{\ell}$ & 0.727 & 0.65 & 0.7 & 0.75 & 0.8 \\
$\mathrm{~S}$ & $L_{s} / L_{\ell}$ & 0.273 & 0.15 & 0.2 & 0.25 & 0.3 \\
\hline
\end{tabular}

Table 2: Effect of factors and contribution ratio

\begin{tabular}{cccccl}
\hline & Level & \multicolumn{4}{c}{ Control Factors } \\
\cline { 3 - 6 } & & $\mathrm{A}$ & $\mathrm{L}$ & $\mathrm{M}$ & $\mathrm{S}$ \\
\hline $\mathrm{SN}$ & 1 & 15.204 & 18.783 & 18.680 & 19.266 \\
& 2 & 18.169 & 18.786 & 18.759 & 18.753 \\
& 3 & 20.344 & 18.780 & 18.761 & 18.606 \\
& 4 & 21.153 & 18.521 & 18.671 & 18.246 \\
\hline $\mathrm{D}$ & & 5.949 & 0.264 & 0.0892 & 1.020 \\
\hline $\mathrm{CR}$ & & 81.24 & 3.61 & 1.22 & 13.93 \\
\hline
\end{tabular}

In order to select the optimum condition, the $\mathrm{SN}$ ratios could be plotted for the four factors (A, L, M, $\mathrm{S})$, as shown in Figure 2. The largest SN ratio of the four levels on each factor might be the best thermal performance, i.e. the optimum condition, as can be conjectured from Eq. (12). As far as the angle was concerned, the angle $20^{\circ}$ was the best for $R_{o}=75,95$, $115 \mathrm{~mm}$ for the increased rate of heat transfer. According to $\mathrm{SN}$ comparisons in Figure 2, the optimum condition was obtained by a combination of levels showing the largest $\mathrm{SN}$ ratio in each control factor, as $\mathrm{A}_{4}, \mathrm{~L}_{2}, \mathrm{M}_{3}$ and $\mathrm{S}_{1}$ for the radius $R_{o}=75,95$, $115 \mathrm{~mm}$.

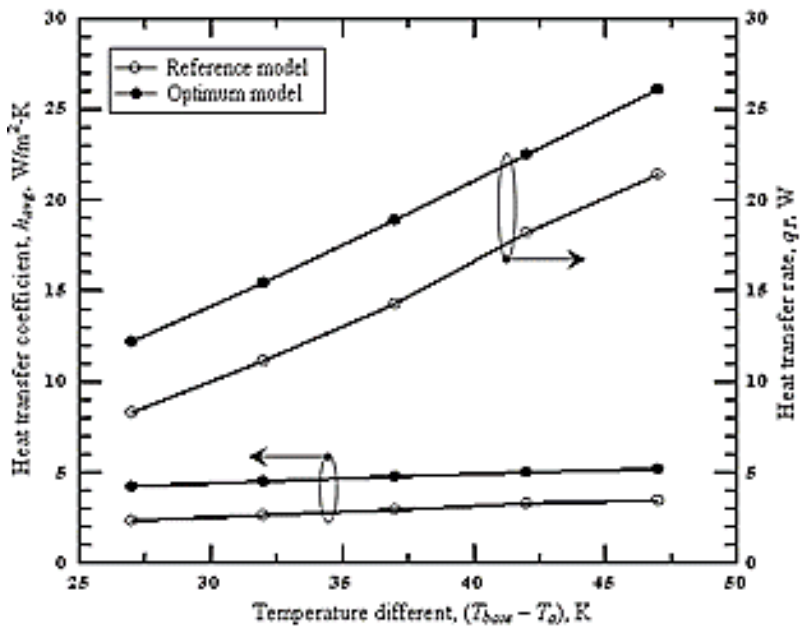

Fig. 2 SN ratio vs control factor

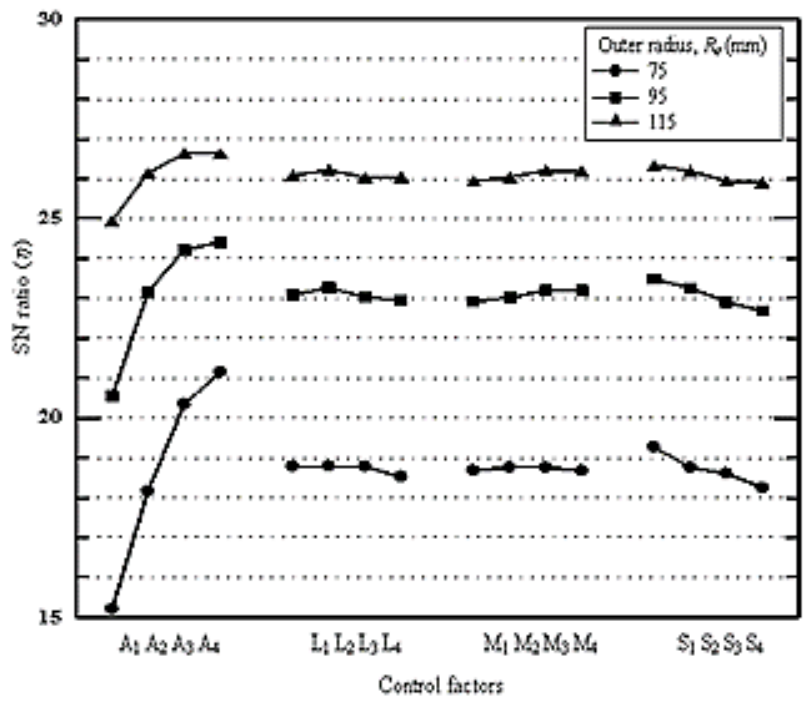

Fig. 3 Heat transfer coefficient vs Temperature difference. 


\subsection{Confirmation test of the optimum model}

The test samples were designed by combining the optimum level on each factor as described above, i.e. $\theta=20^{\circ}, L_{\ell} / R_{o}=0.7, L_{m} / L_{\ell}=0.75$ and $L_{s} / L_{\ell}=0.15$. The heat transfer data was obtained from the sample and the reproducibility of the result was tested as follows for $R_{o}=75 \mathrm{~mm}$.

The presumed SN ratio of the optimum model obtained from Table 2 was compared with the SN ratio of the optimum sample. The presumed SN ratio, $\eta_{\text {pre }}$, was calculated using the $\mathrm{SN}$ ratio corresponding to the optimum level on each factor as $[9,11]$

$$
\begin{aligned}
\eta_{\text {pre }} & =\eta_{A 4}+\eta_{L 2}+\eta_{M 3}+\eta_{S 1}-(m-1) \bar{\eta} \\
& =21.153+18.786+18.761+19.266-3 \times 18.718 \\
& =21.812
\end{aligned}
$$

Here, the values of $\eta_{A 4}, \eta_{L 2}, \eta_{M 3}, \eta_{S I}$ were obtained from Table 2, $m$ indicated the total number of factors, i.e. four, and $\bar{\eta}$ was the average $\mathrm{SN}$ values of the four factors. The $\mathrm{SN}$ ratio of the optimum sample $(\theta$ $=20^{\circ}, L_{\ell} / R_{o}=0.7, L_{m} / L_{\ell}=0.75$ and $\left.L_{s} / L_{\ell}=0.15\right)$ was calculated as 21.724 for $R_{o}=75 \mathrm{~mm}$. This value was in good agreement (within 1\%) with the presumed value in Eq. (13), which ensured the reproducibility of the optimum condition. Similarly, the reproducibility of the optimum test samples for $R_{o}=$ 95 and $115 \mathrm{~mm}$ were tested and obtained good agreement (within $2 \%$ for $R_{o}=95$ and $115 \mathrm{~mm}$ ) with the presumed values.

The average heat transfer coefficient and total heat transfer rate of the optimum model $\left(\theta=20^{\circ}\right.$, $L_{\ell} / R_{o}=0.7, L_{m} / L_{\ell}=0.75$ and $\left.L_{s} / L_{\ell}=0.15\right)$ were compared with the reference model $\left(\theta=18^{\circ}, R_{o}=75\right.$ $\mathrm{mm}, L_{\ell} / R_{o}=0.733, L_{m} / L_{\ell}=0.727$ and $\left.L_{s} / L_{\ell}=0.273\right)$. Figure 3 represents the variations of the average heat transfer coefficient and the total heat transfer rate of the reference model and the optimum model. For $R_{o}=$ $75 \mathrm{~mm}$, the average heat transfer coefficient and the heat transfer rate of the optimum model was higher by $38 \%$ and $23 \%$, respectively than the reference model over the test range $\left(T_{\text {base }}=330 \mathrm{~K}\right.$ to $\left.350 \mathrm{~K}\right)$. It was also obtained that the total heat transfer rate per unit fin area of the optimum model $\left(R_{o}=75 \mathrm{~mm}\right)$ increased of about $40 \%$ than the reference model. Among the optimum models, the heat transfer rate increased of about $26 \%$ and $42 \%$, for $R_{o}=95$ and 115 $\mathrm{mm}$, respectively, than that of $R_{o}=75 \mathrm{~mm}$.

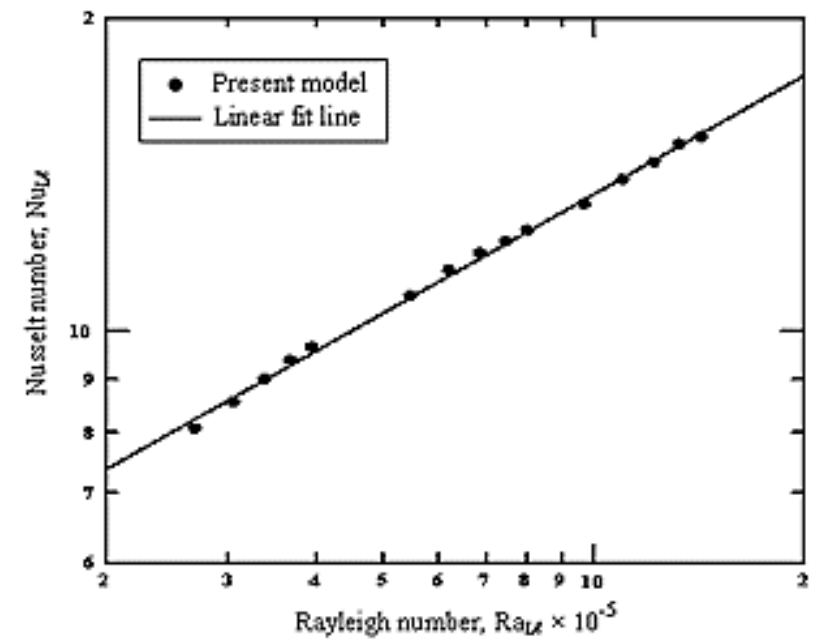

Fig. 4 Nusselt number vs Rayleigh number.

\subsection{Correlation Equation}

It was obtained that the average heat transfer coefficient and the heat transfer rate of the optimum heat sink increased with increasing the outer radius of the heat sink. However, at the optimum condition, the heat transfer data obtained from $R_{o}=75,95$ and $115 \mathrm{~mm}$ could be correlated by an equation. The variation of the Nusselt number $\left(\mathrm{Nu}_{\mathrm{L} \ell}\right)$ with Rayleigh number $\left(\mathrm{Ra}_{\mathrm{L} \ell}\right)$ of the optimum models at different outer radius $(75,95,115 \mathrm{~mm})$ over the test region $\left(T_{\text {base }}=330 \mathrm{~K}\right.$ to $\left.350 \mathrm{~K}\right)$ is shown in Figure 4 . The relation between Nusselt and Rayleigh numbers is given by the linear fit method as

$$
\mathrm{Nu}_{\mathrm{L} \ell}=0.072 \mathrm{Ra}_{\mathrm{L} \ell}{ }^{0.38}
$$

\section{Conclusions}

A steady-state 3D analysis of radial flow heat sink was performed. The model described the effects of various design parameters, namely the lengths of long, medium and short fins, number of fins, and heat sink base radius on heat transfer rate using the Taguchi method. It was obtained that these parameters significantly affected the heat transfer performance of the heat sink. It was also noted that the performance of the optimum heat sink showed superior characteristics compared to the reference heat sink. The design parameters of the optimum heat sink were obtained as $\theta=20^{\circ}, L_{\ell} / R_{o}=0.7, L_{m} / L_{\ell}=0.75$ and $L_{S} / L_{\ell}=0.15$, for the $R_{o}=75,95,115 \mathrm{~mm}$. The factor related to the heat sink surface area, i.e. outer radius, showed significant influence on the heat transfer performance of the heat sink and the heat transfer data at different outer radius were correlated by Eq. (14). These results might be useful to thermal optimization of the radial flow heat sink. 


\section{Nomenclature}

cp

coefficient of heat capacity, $\mathrm{J} /(\mathrm{kg} \cdot \mathrm{K})$

havg heat transfer coefficient, $\mathrm{W} /(\mathrm{m} 2 \cdot \mathrm{K})$

$\mathrm{k}$

kfin

thermal conductivity of air, $\mathrm{W} /(\mathrm{m} \cdot \mathrm{K})$

thermal conductivity of fin, $\mathrm{W} /(\mathrm{m} \cdot \mathrm{K})$

$\mathrm{L} \ell, \mathrm{Lm}, \mathrm{Ls} \quad$ length of long, medium, short fins, $\mathrm{mm}$

Mw the molecular weight of the air

NuLl Nusselt number, havgL $\ell / k$

$\mathrm{n} \ell, \mathrm{nm}, \mathrm{ns} \quad$ no. of the long, medium, short fins

$\mathrm{p} \quad$ Pressure, $\mathrm{Pa}$

$\operatorname{Pr} \quad$ Prandtl number, $v / \alpha$

Rc the universal gas constant

$\mathrm{SN}$ signal-to-noise

RaLe Rayleig number,

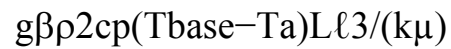

$\mathrm{u}, \mathrm{v}, \mathrm{w} \quad \mathrm{x}, \mathrm{y}, \mathrm{z}$-components of velocity, $\mathrm{m} / \mathrm{s}$

Greek symbols

$\begin{array}{ll}{ }^{\alpha} & \text { thermal diffusivity, } \mathrm{m} 2 / \mathrm{s} \\ \mu & \text { dynamic viscosity, } \mathrm{N} \cdot \mathrm{s} / \mathrm{m} 2 \\ \nu & \text { kinematic viscosity, } \mu / \rho, \mathrm{m} 2 / \mathrm{s} \\ \rho & \text { density of fluid, } \mathrm{kg} / \mathrm{m} 3 \\ \beta & \text { thermal expansion co-efficient, } 1 / \mathrm{K} \\ \theta \mathrm{T} & \text { (Tbase }-\mathrm{Ta}), \mathrm{K}\end{array}$

\section{References}

[1] Kobus, C.J. and Oshio, T. (2005): "Development of a theoretical model for predicting the thermal performance characteristics of a vertical pin-fin array heat sink under combined forced and natural convection with impinging flow". International Journal of Heat and Mass Transfer, Vol. 48, pp. 1053-1063.
[2] Huang, R.T., Sheu, W.J. and Wang, C.C. (2008): "Orientation effect on natural convection performance of square pin fin heat sinks". International Journal of Heat and Mass Transfer, Vol. 51, pp. 2368-2376.

[3] Zografos, A.I. and Sunderland, J.E. (1990): "Natural convection from pin fin arrays". Experimental Thermal and Fluid Science, Vol. 3, pp. 440-449.

[4] Sparrow, E.M. and Vemuri, S.B. (1986): "Orientation effects on natural convection/radiation heat transfer from pinfin arrays". International Journal of Heat and Mass Transfer, Vol. 29, pp. 359-368.

[5] Inada, S., Taguchi, T. and Yang, W.J. (1999): "Effects of vertical fins on local heat transfer performance in a horizontal fluid layer". International Journal of Heat and Mass Transfer, Vol. 42, pp. 2897-2903.

[6] Al-Arabi, M. and El-Riedy, M.K. (1976): "Natural convection heat transfer from isothermal horizontal plates of different shapes". International Journal of Heat and Mass Transfer, Vol. 19, pp. 1399-1404.

[7] Leung, C. W. and Probert, S. D. (1989): "Heat exchanger performance: effect of orientation". Applied Energy, Vol. 33, pp. 235-252.

[8] Taguchi, G. (1991): "Taguchi on robust technology development", Bring Quality Engineering (QE) Upstream ASME.

[9] Taguchi, G., Elsayed, A.E. and Thomas, C.H. (1989), “Quality Engineering in Production Systems", McGraw-Hill, New York.

[10] Lochner J.H. and Matar, J.E. (1990), "Designing for Quality", ASQC Quality Press.

[11] Yun, J.Y. and Lee, K.S. (2000): "Influence of design parameters on the heat transfer and flow friction characteristics of the heat exchanger with slit fins". International Journal of Heat and Mass Transfer, Vol. 43, pp. 2529-2539. 